\title{
Binding indocyanine green to human serum albumin potentially enhances the detection of sentinel lymph nodes. An initial step for facilitating the detection of first-station nodes in penile and other urological cancers
}

\author{
Marcin Markuszewski ${ }^{1}$, Magdalena Buszewska-Forajta ${ }^{2}$, Małgorzata Artymowicz ${ }^{3}$, \\ Wojciech Połom ${ }^{1}$, Marek Roslan ${ }^{4}$, Michał Markuszewski²
}

${ }^{1}$ Department of Urology, Faculty of Medicine, Medical University of Gdansk, Gdansk,
Poland
${ }^{2}$ Department of Biopharmacy and Pharmacokinetics, Faculty of Pharmacy,
Medical University of Gdansk, Gdansk, Poland
${ }^{3}$ Department of Pharmacodynamics, Faculty of Pharmacy, Medical University of Gdansk,
Gdansk, Poland
${ }^{4}$ Department of Urology, Faculty of Medicine, University of Warmia and Mazury, Olsztyn,
Poland

Submitted: 10 June 2019; Accepted: 21 October 2019

Online publication: 8 January 2021

Arch Med Sci 2022; 18 (3): 719-725

DOI: https://doi.org/10.5114/aoms/113237

Copyright @ 2020 Termedia \& Banach

\begin{abstract}
Introduction: Surgical oncology strives to remove the primary cancer tumor together with its local lymphatic tissue. One of the techniques improving the staging of lymph nodes is sentinel node biopsy. The most common agent used in SNB is indocyanine green (ICG). Indocyanine green is characterized by its high affinity for human serum albumin (HSA). In practice, the visualization of the sentinel node is enhanced by attaching a relatively large carrier to the ICG molecule. The aim of this study was to investigate whether the covalent linking of ICG to a nanocolloid would extend the time of detection of the dye as it binds to HSA, assessed by fluorescence measurements in vitro. Material and methods: The influence of the molar concentration of ICG on its ability to form a complex with HSA was investigated. The dye luminescence was measured, with an increasing amount of dye in the presence of a constant concentration of HSA. The stability of the ICG:HSA complex was also investigated.

Results: The binding of ICG and human protein in a solution ratio of $3: 1$ made it possible to detect the ICG luminescence with better and prolonged visibility. In the case of the two lowest ratios, complex formation was not observed. The use of ICG bound to a nanocolloid based on human serum albumin increases the luminescence of the HSA : ICG complex up to $98 \%$. Conclusions: Properly selected proportions of human albumin protein and $\mathrm{ICH}$ allowed higher and longer luminescence to be achieved. Nevertheless, further studies are necessary to establish the optimal concentration ratio.
\end{abstract}

Key words: sentinel lymph node, human serum albumin, indocyanine green, urological cancers.

\section{Introduction}

Indocyanine green (ICG) is a small (776 D), water soluble, anionic, tricarbocyanine dye that can be sterilized, and is safe for use in patients [1].
Corresponding author: Prof. Marek Roslan Department of Urology Faculty of Medicine University of Warmia and Mazury

2 Michała Oczapowskiego St. 10-719 Olsztyn, Poland E-mail: mroslan@gumed.edu.pl 
Because of these and other properties, ICG has been used in clinical medicine since the late 1950s, especially in cardiology, anatomy and physiology [2-4]. Currently, the dye plays a crucial role in modern surgery, especially in visualizing tumors and lymph nodes, as well as improving visualization and rendering the anatomical display more detailed. The need for precise finding of the sentinel nodes seems to be more important in obese patients; the population of them is growing constantly not only in western countries but in Asian countries as well [5].

In urological practice, numerous papers describe the use of ICG for penile, prostate, testicular, renal and bladder cancers [6-10]. Cancers of other organs are also involved in migrating to sentinel nodes [11]. In fact, there are no standards for indicating migration to sentinel nodes for the most common urological cancers such as bladder and renal cancers because they spread not only via lymphatic vessels. Notably, the spread of neoplastic cells seems to be more predictable in penile cancer; thus, it is particularly important to find sentinel nodes in this tumor [12]. A significant feature of ICG is its high sensitivity to light, and its low stability in aqueous solution $[3,6]$.

The most essential aim of contemporary oncological surgery is the radical excision of neoplastic tissues. The main route of cancer cell migration is the lymphatic system. The standard surgical procedure consists of tumor resection and removal of local lymph nodes that are potential sites of cancer cell migration. This method increases the chances of the radical removal of the tumor, but is simultaneously burdened with the risk of unnecessary excision of healthy tissue and the occurrence of complications [13]. To improve the staging of the disease, it is crucial to identify the sentinel node, which is considered to be the first site of metastasis. However, the appropriate identification of the sentinel node is still a challenging problem [14].

One of the new options is to use fluorescence-guided surgery for sentinel node biopsy [15]. When the sentinel node is not metastatic, there is no need for the excision of other lymph nodes, which is also beneficial, since the removal of a single lymph node is usually significantly easier and less invasive. The use of a specific dye, such as ICG, facilitates the localization of the sentinel node. ICG can be injected into the tissues surrounding the tumor as a complex with human serum albumin (HSA), which stabilizes the dye molecules as well as potentiating its fluorescence $[1,16,17]$.

According to van der Poel et al. [7], ICG was introduced as a complex composed of the dye molecules and a nanocolloid of HSA, where the ICG : HSA molar ratio was $40: 1$ [18]. The protocol provided information that the dye binds to the HSA molecule within $1 \mathrm{~min}$, and that its concentration is much higher compared to the HSA only [18]. In this case, the use of a molar composition provides the tissue contamination; thus, clear visualization of the tumor area can become problematic. For this reason, there is a need to identify the optimal ICG : HSA concentration ratio that provides the highest tumor fluorescence with concomitant reduced interference by unbound molecules of ICG. The main aim of the project was to perform an in vitro study that can define the molar composition of the ICG:HSA complex that would enable the achievement of the highest fluorescence with only a trace concentration of unbound ICG. We determined the complexed ICG, using highperformance liquid chromatography, coupled with a fluorescence detector (HPLC-FLD).

A significant difficulty in using dyes is their rapid passage through the targeted sentinel nodes, and their accumulation in other lymph nodes. In some papers, the authors reported evidence that binding of ICG to HSA may be helpful in achieving prolonged accumulation in the sentinel nodes $[17,19]$.

The goal of this study was to define the optimal molar ratio of ICG to HSA in vitro that would produce the highest fluorescence, which could be translated to clinical use during sentinel lymph node biopsy.

\section{Material and methods}

\section{Materials}

\section{Chemicals}

Human serum albumin (HSA; Nano-Albumin $1 \mathrm{mg}$ ) was purchased from MediRadiopharma Ltd. (Hungary). Indocyanine green (ICG-Pulsion) was obtained from Pulsion Medical Systems SE (Munich, Germany). Water was purified in a Millipore Direct-Q 3 UV Water Purification System (Millipore Corporation, Bedford, MA, USA).

\section{Apparatus}

The analyses were performed with a high performance liquid chromatography system (HPLC, 1200) with a fluorescence detector (FLD, 1290) from Agilent Technologies (Palo Alto, CA, USA).

\section{Methods \\ Sample preparation}

\section{Albumin solution}

A stock solution of albumin $(1 \mathrm{mg} / \mathrm{ml})$ was prepared as follows: $1 \mathrm{mg}$ of albumin was dissolved in $1 \mathrm{ml}$ of purified water and vortex-mixed ( 2 min, RT). Next, $50 \mu$ l of HSA stock solution was diluted in purified water to a final concentration of $5 \mu \mathrm{g} / \mathrm{ml}$. 
Binding indocyanine green to human serum albumin potentially enhances the detection of sentinel lymph nodes. An initial step for facilitating the detection of first-station nodes in penile and other urological cancers

Table I. Volumes of components used for the preparation of ICG : HSA complex solution

\begin{tabular}{|c|c|c|c|c|c|}
\hline \multirow{2}{*}{$\begin{array}{l}\text { ICG : HSA } \\
\text { complex }\end{array}$} & \multicolumn{3}{|c|}{ ICG solution } & \multirow{2}{*}{$\begin{array}{c}\text { HSA solution } \\
5 \mu \mathrm{g} / \mathrm{ml}\end{array}$} & \multirow{2}{*}{$\begin{array}{l}\text { Purified water } \\
\qquad[\mu \mathrm{l}]\end{array}$} \\
\hline & $5 \mu \mathrm{g} / \mathrm{ml}$ & $2.5 \mu \mathrm{g} / \mathrm{ml}$ & $0.25 \mu \mathrm{g} / \mathrm{ml}$ & & \\
\hline $1: 1$ & - & - & 24.0 & 100 & 76.0 \\
\hline $2.6: 1$ & - & - & 62.4 & 100 & 37.6 \\
\hline $5.3: 1$ & - & 12.5 & - & 100 & 87.5 \\
\hline $7.5: 1$ & - & 17.5 & - & 100 & 82.5 \\
\hline $10.7: 1$ & 12.5 & - & - & 100 & 87.5 \\
\hline $21.4: 1$ & 25.0 & - & - & 100 & 75.0 \\
\hline $32.1: 1$ & 37.5 & - & - & 100 & 62.5 \\
\hline $42.8: 1$ & 50.0 & - & - & 100 & 50.0 \\
\hline
\end{tabular}

ICG - indocyanine green, HSA - human serum albumin.

Table II. Number of moles of ICG and HSA in every ratio of complex composition

\begin{tabular}{|c|c|c|c|c|}
\hline ICG : HSA ratio & Mass of ICG [ng] & Number of moles of ICG & Mass of HSA [ng] & Number of moles of HSA \\
\hline $1: 1$ & 6.0 & $7.74 \times 10^{-12}$ & 500 & $7.52 \times 10^{-12}$ \\
\hline $2.6: 1$ & 15.6 & $20.13 \times 10^{-12}$ & 500 & $7.52 \times 10^{-12}$ \\
\hline $5.3: 1$ & 31.3 & $40.32 \times 10^{-12}$ & 500 & $7.52 \times 10^{-12}$ \\
\hline $7.5: 1$ & 43.8 & $56.45 \times 10^{-12}$ & 500 & $7.52 \times 10^{-12}$ \\
\hline $10.7: 1$ & 62.5 & $80.64 \times 10^{-12}$ & 500 & $7.52 \times 10^{-12}$ \\
\hline $21.4: 1$ & 125.0 & $161.29 \times 10^{-12}$ & 500 & $7.52 \times 10^{-12}$ \\
\hline $32.1: 1$ & 187.5 & $241.94 \times 10^{-12}$ & 500 & $7.52 \times 10^{-12}$ \\
\hline $42.8: 1$ & 250.0 & $322.59 \times 10^{-12}$ & 500 & $7.52 \times 10^{-12}$ \\
\hline
\end{tabular}

ICG - indocyanine green, HSA - human serum albumin.

\section{Indocyanine green solution}

A stock solution of ICG $(1 \mathrm{mg} / \mathrm{ml})$ was prepared as follows: $1 \mathrm{mg}$ of ICG was dissolved in $1 \mathrm{ml}$ of purified water and vortex-mixed for 2 min at RT. The stock solution was diluted in purified water to obtain the following concentrations: $5 \mu \mathrm{g} / \mathrm{ml}$, $2.5 \mu \mathrm{g} / \mathrm{ml}, 0.25 \mu \mathrm{g} / \mathrm{ml}$.

\section{ICG : HSA complexes}

Solutions of the ICG : HSA complex were prepared at eight molar ratios (ICG : HSA): 1 : 1 , $2.6: 1 ; 5.3: 1 ; 7.5: 1 ; 10.7: 1 ; 21.4: 1 ; 32.1: 1$ and $42.8: 1$. The following tables present the component volumes used for the preparation of the ICG : HSA complex solutions (Table I) and the number of moles of ICG and HSA at every ratio (Table II).

\section{ICG : HSA complex filtration}

Two hundred microliters of every ratio of ICG: HSA complex were injected into Vivaspin 2 Concentrators (Sartorius) with poly-ether sulfonate membranes $(10,000$ MWCO) in order to separate the complexes from the unbound fraction of ICG. Samples were centrifuged for $5 \mathrm{~min}$ at $4^{\circ} \mathrm{C}$. The filtrate was collected and analyzed with the same analytical method.

\section{High-performance liquid chromatography analyses}

ICG was measured by a HPLC-FLD technique, with a 3 min analysis run time. Water with flow $0.5 \mathrm{ml} / \mathrm{min}$ was used as mobile phase. Injection volume was $2 \mu \mathrm{l}$. Three sets of samples were prepared: solutions of ICG, complexes of ICG : HSA and filtrates obtained with Vivaspin concentrators. Fluorescence was measured at $314 \mathrm{~nm}$, with an excitation wavelength of $270 \mathrm{~nm}$.

\section{Results}

\section{Analytical method development and optimization}

The analytical method was optimized according to mobile phase composition and flow, injec- 
A

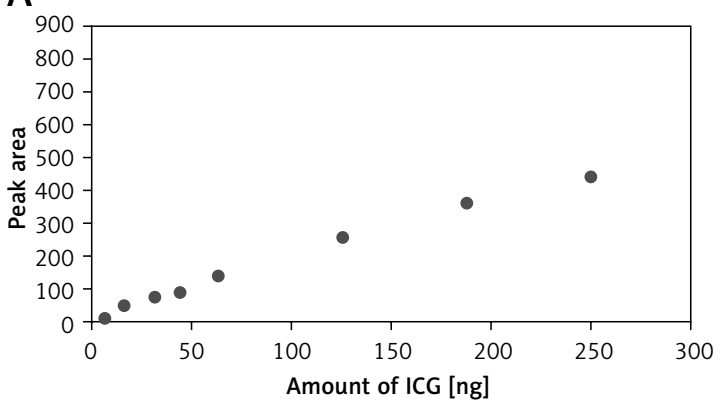

B

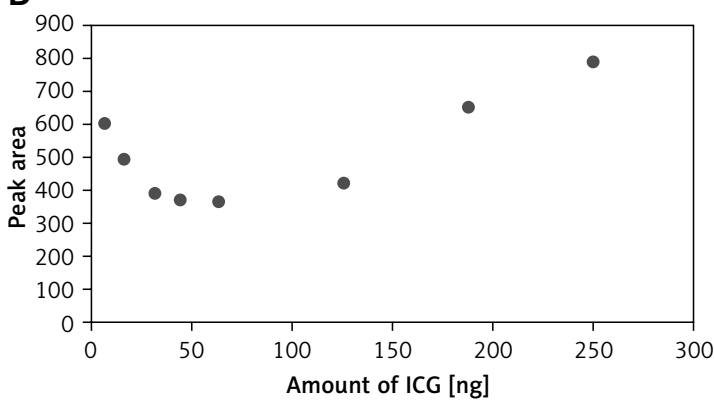

C

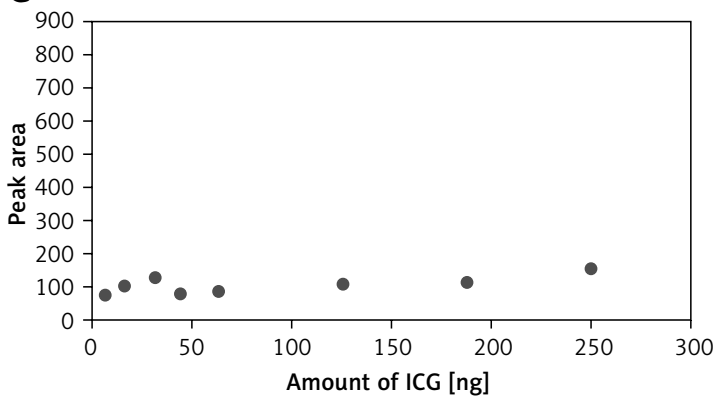

Figure 1. Peak area as a function of the indocyanine green (ICG) amount obtained for a pure solution of ICG (A), the ICG : HSA complex in solution (B), and cut-off filtrate (C)

tion volume and excitation/emission wavelength, where the following magnitudes were checked: $214 \mathrm{~nm} / 370 \mathrm{~nm}$ and $270 \mathrm{~nm} / 314 \mathrm{~nm}$. As a result, the following parameters were chosen: water as mobile phase with a flow rate of $0.5 \mathrm{ml} / \mathrm{min}$, injection volume of $2 \mathrm{ml}$, and $270 \mathrm{~nm} / 314 \mathrm{~nm}$ as the excitation and emission wavelengths. Optimization of the parameters enabled measurements with the highest sensitivity within a relatively short period of time. A separation column was not used, because omission of the separation process better simulated physiological conditions.

\section{In vitro study}

Three sets of samples were prepared. First, color changes, transparency, and formation of sediment were assessed visually in ICG : HSA mixtures. In the second step, ICG was quantified using HPLCFLD to select the ICG : HSA ratio with the lowest amount of free ICG. For this reason, three types of samples for each studied concentration of ICG were measured:

1 ) the aqueous solution of ICG (dye mass ranging from $6 \mathrm{ng}$ to $250 \mathrm{ng}$ ),

2) the solution of ICG : HSA complex (ICG mass ranging from $6 \mathrm{ng}$ to $250 \mathrm{ng}$, with the HSA mass at $500 \mathrm{ng}$ ),

3) the filtrate (ICG mass ranging from 6 ng to $250 \mathrm{ng}$ and the HSA mass at $500 \mathrm{ng}$ ), following filtration on Vivaspin 2 Centrifugal Concentrators after preparation of the ICH : HSA complex.

\section{Aqueous solutions of indocyanine green}

The fluorescence of samples was analyzed at eight different molar ratios. In case of the aqueous solution of ICG, the method demonstrated a linear response from 6 to $250 \mathrm{ng}$ dye, with R2 greater than 0.9915 . In practice, this means that the fluorescence of a pure ICG solution increases with the increase in ICG concentration. The highest fluorescence was observed with 250 ng of ICG (Figure 1 A).

\section{Aqueous solutions of the ICG : HSA complex}

According to clinical observations, an ICG : HSA complex solution with excess free ICG leads to the indiscriminate staining of many lymph nodes, following injection to the patient. The following in vitro studies were carried out to select the complex composition that provides the highest fluorescence arising from the complex, with the lowest fluorescence originating from the free dye. The main goal of this part of the study was to analyze the influence of the ICG concentration on ICG : HSA complex formation. The highest molar ratio was selected in reference to the protocol proposed by Brouwer et al. [12]. The results indicate that there is no linear response over the dye amount in the complex ranging from 6 to $250 \mathrm{ng}$. The response can be characterized by an exponential function, which means that the fluorescence decreases as the molar ratio increases up to $5.3: 1$ (ICG : HSA). In the case of $7.5: 1$, a minimum in fluorescence was observed and was stable up to the 10 : 1 (ICG : HSA) molar ratio. Then, the concentration increased with an increasing amount of ICG. The results are presented in Figure $1 \mathrm{~B}$.

\section{The filtrate of the aqueous solution of the ICG:HSA complex}

The fluorescence of the filtrate was relatively high at the lowest molar ratio. Then, the fluorescence decreased as the molar ratio increased up to 5.3 : 1 (ICG : HSA). At the 7.5 : 1 (ICG : HSA) molar ratio, the fluorescence was reduced, and stable up to the 10: 1 molar ratio. From this point, the fluorescence increased slightly with the increasing amount of IC (Figure $1 \mathrm{C}$ ). 


\section{Discussion}

Currently available methods of visualization of the sentinel node are based on the use of radioactive complexes. Although the visualization is satisfactory, the procedure using radioactive complexes needs to be performed in a special nuclear medicine facility. One of the widely applied methods is the use of the radioactive complex of technetium and nanocolloid of albumin, visible in spectral CT. Detection of nodes during an operation is carried out using a gamma camera, which is expensive and technically challenging.

Indocyanine green seems to have many advantages as a simple method of adding markers and easy identification of the sentinel node by intraoperative surgery using an infrared camera. Indocyanine green is readily available, cheap and above all safe, although the depth of visualization and higher number of stained lymph nodes are the major weak points of this method.

Our results showed that the quality of visualization is strictly correlated with the ratio of HSA and ICG. In case of excess ICG, free dye may be observed. Unbound ICG has a high affinity for biological barriers, which would result in vague images of the sentinel node, as shown by the results of Kuzmin et al. and van der Poel et al. [17, 19]. However, they did not study whether complexation of ICG with a protein may be superior to pure ICG in diagnosing sentinel nodes. This encouraged us to investigate whether the combination of ICG with HSA would improve the detection of lymph nodes involved in cancer.

In reference to the theory of complexation, complex formation includes three physiochemical basic steps - association of the particles with the surface, transfer of the molecule from the surface and eventual absorption into the target molecule.

Our results show that the $1: 1$ (ICG : HSA) complex has a relatively high fluorescence that decreases with increase of the molar ratio up to 7.5 : 1 corresponding to $43.75 \mathrm{ng}$ ICG. The measured fluorescence is mainly of HSA origin, which indicates that the complex is not created or created only with limited efficiency as a result of a limited amount of dye. Moreover, any small amount of the dye that may have bound would only suppress the fluorescence originating from HSA.

In the case of the complex, minimal fluorescence is observed for both $7.5: 1$ and 10.7: 1 (ICG : HSA) molar ratios. Finally, with an increasing amount of ICG, the fluorescence of the complex also increases. Based on these results, one can assume that a small amount of dye does not lead to complexation. However, when the optimum molar ratio is obtained (minimum molar ratio of $7.5: 1$ (ICG : HSA)), the complex is created, and from this point on, increasing the amount of ICG leads to the enhancement of complex fluorescence. To prove this hypothesis the following studies were conducted. The fluorescence of pure ICG and that of pure HSA were measured, and were used to perform the calculation of relative fluorescence of the complex. For this purpose, on the one hand, the fluorescence of the complex was measured in reference to the initial fluorescence of the ICG aqueous solution at an adequate concentration. On the other hand, the fluorescence of the complex was calculated in reference to the initial fluorescence of the HSA aqueous solution (Table III).

Binding of the ICG to the HSA was assessed by measuring the free ICG. For this purpose, samples were prepared according to the above-mentioned protocols, and filtered using selective membranes (cut-off $=10 \mathrm{kDa}$ ) that should retain $99 \%$ of the albumin in free and ICG-bound form. Thus, it was assumed that the filtrate contains only free ICG.

In the range $1: 1$ to $5.3: 1$ (ICG : HSA) molar ratio, the fluorescence of the filtrate samples increased, which was caused by the association of trace amounts of ICG molecules with HSA. Moreover, the increased fluorescence of free ICG can be explained by the dissociation of the dye dimers and aggregates. This phenomenon is in accordance with the decreasing fluorescence of the complex with molar composition from $1: 1$ to $5.3: 1$ (ICG : HSA).

Complexes with the molar ratios $7.5: 1$ and 10.7 : 1 had minimal fluorescence. In these cases, $80 \%$ of ICG is bound to HSA; therefore, the filtrate contains only a trace concentration of ICG. Increasing the amount of ICG from this ratio causes the enhancement of complex fluorescence. This is a consequence of the increasing amount of ICG, which is at a much higher concentration compared to that of HSA.

In surgical practice, a highly significant issue is to limit the operation to the smallest possible range [20]. In cancer surgery, decreasing the number of removed nodes may result in a smaller number of complications, which cause greater morbidity if they appear. The potential significance of this study is defining the optimum ICG/ HSA molar ratio that allows extension of the time of detection and increases the intensity of fluorescence. Nevertheless, the results of our study may help the surgeon in excision of sentinel nodes and make the surgery more efficient and less invasive at the same time. We consider the results of this paper as the first step for further development of this technique. If this goal were achieved, it is likely that more urologists would introduce the technique into standard practice.

The strengths of the present study include the finding that the optimal ICG to HSA ratio of $7.5: 1$ to $10.5: 1$ increases the fluorescence 
Table III. Calculation of fluorescence in reference to aqueous solution of pure ICG (A) and aqueous solutions of pure HAS (B)

A

\begin{tabular}{|lccccc|}
\hline Molar ratio of ICG : HSA & Amount of ICG [ng] & \multicolumn{2}{c}{ ICG } & \multicolumn{3}{c|}{ Complex ICG : HSA } \\
\cline { 3 - 6 } & & Fluorescence [RFU] & $(\%)$ & Fluorescence & (\%) \\
\hline $1: 1$ & 6.00 & 13 & 100 & 604 & 4646 \\
\hline $2.6: 1$ & 15.60 & 78 & 100 & 501 & 642 \\
\hline $5.3: 1$ & 31.25 & 57 & 100 & 396 & 694 \\
\hline $7.5: 1$ & 43.75 & 97 & 100 & 375 & 386 \\
\hline $10.7: 1$ & 62.50 & 146 & 100 & 373 & 255 \\
\hline $21.4: 1$ & 125.00 & 262 & 100 & 426 & 163 \\
\hline $32.1: 1$ & 187.50 & 365 & 100 & 658 & 180 \\
\hline $42.8: 1$ & 250.00 & 435 & 100 & 794 & 183 \\
\hline
\end{tabular}

B

\begin{tabular}{|lccccc|}
\hline Molar ratio of ICG : HSA & Amount of HSA [ng] & \multicolumn{2}{c}{ HSA } & \multicolumn{2}{c|}{ Complex ICG : HSA } \\
\cline { 2 - 6 } & & 661 & 100 & 604 & 91 \\
\hline $1: 1$ & 500 & 661 & 100 & 501 & 76 \\
\hline $2.6: 1$ & 500 & 661 & 100 & 396 & 60 \\
\hline $5.3: 1$ & 500 & 661 & 100 & 375 & 57 \\
\hline $7.5: 1$ & 500 & 661 & 100 & 373 & 56 \\
\hline $10.7: 1$ & 500 & 661 & 100 & 426 & 64 \\
\hline $21.4: 1$ & 500 & 661 & 100 & 658 & 99 \\
\hline $32.1: 1$ & 500 & 661 & 100 & 794 & 120 \\
\hline $42.8: 1$ & 500 & & & & \\
\hline
\end{tabular}

ICG - indocyanine green, HSA - human serum albumin.

of the bound molecule and decreases the amount of free ICG. Therefore, this result can be a potential factor for better visualization of sentinel nodes and less contamination of other nodes. The potential significance of this study is defining the proportion of the ICG/HSA molecule that allows the time of detection to be extended and increases the intensity of fluorescence. If this goal were to be achieved, more urologists would introduce the technique into standard practice.

There are some limitations of this study that should be recognized and discussed. The preclinical nature of the study is an important limitation, because it introduces some difficulties in defining a standard response to each dose of ICG. Moreover, we are not in a position to determine how well the results of our study can be translated to a population of patients undergoing cancer surgery. To facilitate this translation, we first plan to apply our method of optimal proportion of ICG/ HSA in a porcine model.
In future experiments, we would like to show how the new complexation concentrations of ICG with HSA affect uptake or visualization in lymph nodes, as well as in the setting of lymphography for urologic-based cancers. The initial animalbased studies will be performed to show lymph node localization or differential levels of detection. Moreover, further investigations on the presented complexes of ICG/HSA might be verified in performing sentinel node excision via minimally invasive procedures, including laparoscopy or laparoendoscopic single-site surgery [21].

In conclusion, the use of ICG bound with a human serum protein increases the luminescence of ICG nodes up to $98 \%$. Increasing the amount of ICG has the effect of increasing the luminescence of the ICG solution. This result suggests that the proportion of $7.5: 1$ to $10.5: 1$ (ICG : HSA) can provide more satisfactory visualization of sentinel nodes, thereby helping the surgeon with their excision. However, it is worth noting that for better 
understanding of the molecular mechanism, as well as clinical usefulness, further investigations through in vitro and in vivo studies are needed.

\section{Conflict of interest}

The authors declare no conflict of interest.

\section{References}

1. Boni L, David G, Mangano A, et al. Clinical applications of indocyanine green (ICG) enhanced fluorescence in laparoscopic surgery. Surg Endosc 2015; 29: 2046-55.

2. Baillif S, Wolff B, Paoli V, et al. Retinal fluorescein and indocyanine green angiography and spectral-domain optical coherence tomography findings in acute retinal pigment epitheliitis. Retina 2011; 31: 1156-63.

3. Mordon S, Devoisselle JM, Soulie-Begu S, Desmettre T. Indocyanine green; physiochemical factors affecting its fluorescence in vivo. Microvasc Res 1998; 55: 146-52.

4. Noura S, Ohue M, Seki Y, et al. Feasibility of a lateral region sentinel node biopsy of lower rectal cancer guided by indocyanine green using a near-infrared camera system. Ann Surg Oncol 2010; 17: 144-51.

5. Mazidi $M$, Banach $M$, Kengne $A P$, et al. Prevalence of childhood and adolescent overweight and obesity in Asian countries: a systematic review and meta-analysis. Arch Med Sci 2018; 14: 1185-1203.

6. Markuszewski M, Polom W, Cytawa W, et al. Comparison of real-time fluorescent indocyanine green and $(99 \mathrm{~m})$ Tc-nanocolloid radiotracer navigation in sentinel lymph node biopsy of penile cancer. Clin Genitourin Cancer 2015; 13: 574-80.

7. van der Poel HG, Buckle T, Brouwer OR, et al. Intraoperative laparoscopic fluorescence guidance to the sentinel lymph node in prostate cancer patients: clinical proof of concept of an integrated functional imaging approach using a multimodal tracer. Eur Urol 2011; 60: 826-33.

8. Brouwer OR, Valdés Olmos RA, Vermeeren L, et al. SPECT/CT and a portable gamma-camera for imageguided laparoscopic sentinel node biopsy in testicular cancer. J Nucl Med 2011; 52: 551-554.

9. Bex A, Vermeeren L, de Windt G, et al. Feasibility of sentinel node detection in renal cell carcinoma: a pilot study. Eur J Nucl Med Mol Imaging 2010; 37: 1117-23.

10. Schaafsma BE, Verbeek FP, Elzevier HW, et al. Optimization of sentinel lymph node mapping in bladder cancer using near-infrared fluorescence imaging. J Surg Oncol 2014; 110: 845-50.

11. Kaczka KA, Pomorski L. One-step nucleic acid amplification analysis of sentinel lymph nodes in papillary thyroid cancer patients. Arch Med Sci 2017; 13: 1416-26.

12. Brouwer R, van der Poel G, Bevers RF, et al. Beyond penile cancer, is there a role for sentinel node biopsy in urological malignancies? Clin Transl Imaging 2016; 4: 395-410.

13. Valero MG, Golshan M. Management of the axilla in early breast cancer. Cancer Treat Res 2018; 173: 39-52.

14. Moncayo VM, Alazraki AL, Alazraki NP, Aarsvold JN. Sentinel lymph node biopsy procedures. Semin Nucl Med 2017; 47: 595-617.

15. Reinhart MB, Huntington CR, Blair LJ, et al. Indocyanine green: historical context, current applications, and future considerations. Surg Innov 2016; 23: 166-75.

16. Curry S, Mandelkow H, Brick P, Franks N. Crystal structure of human serum albumin complexed with fatty acid reveals an asymmetric distribution of binding sites. Nat Struct Biol 1998; 5: 827-35.

17. Kuzmin VA, Podrugina TA, Golovina GV, et al. Complex formation of albumin with tricarbocyanine dyes containing phosphonate groups. Photochem Photobiol Sci 2016; 15: 1377-84.

18. Brouwer OR, Buckle T, Vermeeren L, et al. Comparing the hybrid fluorescent-radioactive tracer indocyanine green with $99 \mathrm{mTc}$-nanocolloid for sentinel node identification: a validation study using lymphoscintigraphy and SPECT/CT. J Nucl Med 2012; 53: 1034-40.

19. van der Poel HG, Buckle T, Brouwer OR, et al. Intraoperative laparoscopic fluorescence guidance to the sentinel lymph node in prostate cancer patients: clinical proof of concept of an integrated functional imaging approach using a multimodal tracer. Eur Urol 2011; 60: 826-33.

20. Aoun F, Albisinni S, Zanaty M, et al. Indocyanine green fluorescence-guided sentinel lymph node identification in urologic cancers: a systematic review and meta-analysis. Minerva Urol Nefrol 2018; 70: 361-9.

21. Roslan M, Markuszewski M, Kłącz J, et al. Laparoscopic nephroureterectomy with transvesical single-port distal ureter and bladder cuff dissection: points of technique and initial surgical outcomes with five patients. Videosurgery Miniinv 2014; 9: 267-72. 